\title{
Diverse virulence traits underlying different clinical outcomes of Salmonella infection
}

\author{
Joshua Fierer ${ }^{1,2,3}$ and Donald G. Guiney ${ }^{1}$ \\ ${ }^{1}$ Department of Medicine, and \\ ${ }^{2}$ Department of Pathology, University of California at San Diego School of Medicine, La Jolla, California, USA \\ ${ }^{3}$ Veterans Affairs San Diego Healthcare System, San Diego, California, USA
}

Address correspondence to: Joshua Fierer, Veterans Affairs San Diego Healthcare System, Division of Infectious Diseases (111F), 3350 La Jolla Village Drive, San Diego, California 92161, USA. Phone: (858) 552-7446; Fax: (858) 552-4398; E-mail: jfierer@ucsd.edu.

Nontyphoid Salmonella are a common cause of bacterial gastroenteritis, which is usually a self-limited illness in previously healthy adults (1). However, nontyphoid Salmonella can also cause a variety of life-threatening extraintestinal infections. A number of host factors can predispose to extraintestinal Salmonella infections, including AIDS, hemolytic anemias, and genetic defects that affect IL-12, IFN- $\gamma$, and the phagocyte NADPH oxidase, but even in the susceptible host, certain serotypes of Salmonella are more likely to cause systemic infections. These same serotypes are also more likely to cause invasive infections in people with no identifiable predisposing abnormalities. This review will concentrate on the differences among nontyphoid Salmonella that could account for the increased virulence of some serotypes or strains.

\section{Phenotypic and genetic differences among Salmonella strains}

Based on iso-enzymes, rRNA sequences, and DNA hybridization, microbial taxonomists consider almost all of the Salmonella that infect mammals and birds to be one species (S. enterica) (2). There are six subspecies of S. enterica including enterica (I), salamae (II), arizonae (IIIa), diarizonae (IIIb), houtenae (IV), and indica (VI). S. bongori $(\mathrm{V})$ is rarely isolated from clinical specimens and is considered to be a separate species (see below). Within these six subspecies are over 2,000 serotypes of Salmonella, as defined by the carbohydrate structures found in LPS and by flagella antigens. However, nearly all the serotypes that cause disease in humans and domestic animals belong to subspecies enterica (I).

Despite the fact that they are genetically closely related, there is enormous variation in the virulence and epidemiology of different serotypes of S. enterica (I). For instance, most serotypes cause only gastroenteritis, while specific serotypes (S. typhi, S. paratyphi A and C, and $S$. sendai) cause enteric fever, and a few nontyphoid serovars, e.g., S. cholerae-suis and S. dublin, are more likely to cause bacteremia than diarrhea (3). Likewise, some Salmonella are either fully host-adapted (as in the case of S. typhi and S. pullorum, which are limited to people and chickens, respectively) or highly host adapted, as with S. cholerae-suis, S. abortus-ovis, and S. dublin, which are typically associated with swine, sheep, and cattle, respectively. The grouping together of organisms with very different invasive potentials into the species $S$. enterica obscures important clinical, epidemiological, and biological differences among the serotypes, which is an important reason to retain the distinctions that are now relegated to serovar designations.

The biological and genetic mechanisms underlying the phenotypic diversity of Salmonella strains are beginning to be understood. Genetic variations, or polymorphisms, are particularly prominent in two general classes of loci: (a) genes encoding surface structures such as LPS, flagella, and fimbriae, and (b) specific virulence genes encoding factors that modify host cell physiology or protect the bacteria from the antimicrobial systems of the host. The surface structures not only affect the virulence of the bacteria, but also are key targets of the host immune system, resulting in selective pressure to generate genetic polymorphisms coding for antigenic diversity. In addition, specific virulence determinants may be clustered together on polymorphic pathogenicity islands or located on transmissible genetic elements such as plasmids or phage - all arrangements that facilitate the modular transmission of genes involved in pathogenesis and thus increase the diversity in virulence phenotypes among strains.

\section{Surface structures}

LPS. The genes encoding the enzymes that synthesize the sugars and organize them into the polysaccharide sidechains on the LPS are clustered together at the $r f b$ locus on the chromosome. The Kauffmann-White scheme that is used to serotype Salmonella (as well as Escherichia coli and other strains; see Donnenberg and Whittam, this series, ref. 4) is based on antigenic polymorphisms of LPS $(\mathrm{O})$ and flagella $(\mathrm{H})$. Although the core structure of the LPS (lipid A) is largely conserved in all Salmonella, the polysaccharide side chains are highly polymorphic because the $r f b$ region is polymorphic (5). Classically, the antibodies used to group salmonellae are raised in rabbits that have been immu- 
nized with heat-killed bacteria. The exact structure of many of the carbohydrate antigens is not known and may be difficult to discern, because epitopes found on helical polysaccharides may not be linear (6).

Almost all human infections (>95\%) are caused by Salmonella that are in groups (defined by $\mathrm{O}$ antigen structures) A, B, C, D, and E. Interestingly, groups A, B, and $\mathrm{D}$ have the same common trisaccharide backbone structure of their LPS: D mannose $\alpha 1 \rightarrow 4$ L rhamnose $\beta 1 \rightarrow 3$ D galactose $\alpha 1 \rightarrow 2$. The only difference between the three LPS structures is the di-deoxyhexose that is attached to the 3 position of the mannose. In group $\mathrm{A}$ the di-deoxyhexose is paratose, in B it is abequose, and in D it is tyvelose. In group E Salmonella, the backbone structure is made up of the same trisaccharide, but there is no di-deoxyhexose. The group C LPS structure is made up of a linear polymer of four mannose residues and one $\mathrm{N}$-acetyl glucosamine residue.

Although a few serotypes of Salmonella have an outer capsular layer (notably S. typhi), in most serotypes LPS forms the layer around the bacterium that protects it from the environment. LPS interacts with both antibodies and with complement. Rough Salmonella mutants, which lack this protective layer, activate complement by the alternative pathway, leading to killing of the bacteria by the membrane attack complex of complement. Not surprisingly, rough mutants are avirulent. Naturally occurring infections are caused by Salmonella with a complete LPS, and they are resistant to killing by complement (7). However, activated complement protein C 3 covalently binds to the terminal sugars on the LPS and acts as an opsonin. In the case of groups A, B, and D Salmonella, these terminal sugars are di-deoxyhexoses, which therefore modulate the interaction with complement (C3) (8). Changing the structure of the di-deoxyhexose from tyvelose to abequose increases the amount of $\mathrm{C} 3$ that binds to the bacteria, which in turn increases phagocytosis by macrophages (9). This change also affects the virulence of these bacteria in mice (10), which is due largely to differences in phagocytosis and killing by polymorphonuclear leukocytes (J. Fierer, unpublished observations). It is clearly not true that group B salmonellae are more virulent in humans, as S. typhi is a group D Salmonella. Human complement is much more active than mouse complement, which may explain why $\mathrm{C} 3$ activation and binding is a limiting step in mice but not humans.

Much of the antibody response to Salmonella infection is directed against the LPS, and especially against the dideoxyhexoses, if they are present. The terminal sugar fits into the antigen binding site of the antibody, where hydrogen bonds form between aromatic amino acids and the $\mathrm{OH}$ groups on the di-deoxyhexose (11). Although the relative importance of antibodies and $\mathrm{T}$ lymphocytes in suppressing Salmonella infections is controversial (12), there is ample evidence that antibodies protect both humans and mice against Salmonella (13, 14). Furthermore, in most experimental systems, immunity to Salmonella is $\mathrm{O}$ antigen-specific $(15,16)$. Thus, it might benefit the bacteria to change the structure of their LPS, which is indeed observed, although not during the course of a single infection. O-antigen variation between isolates can occur because of lysogenic conversion by bacteriophages or mutations in chromosomal genes. Most of the variants are O-acetylated or glucosylated sugars, which can change the LPS structure enough to create new antigens. For instance, OafA is a chromosomal enzyme that O-acetylates abequose creating a new antigen (O5) (17). There is a phage that adds glucose to some of the galactose residues on the repeating trisaccharide unit in $S$. typhimurium which creates antigen $\mathrm{O} 1$, a common alteration. Phages can also change the linkages between the sugars. None of these substitutions is ever stoichiometric, so both the original and the modified LPS are expressed on the same bacteria. Very little is known about the regulation of these enzymatic modifications.

Because the antibody response is largely directed toward the $\mathrm{O}$ antigen, the polymorphism of $\mathrm{O}$ antigens could allow Salmonella that modify these antigens to infect hosts that already have antibody to unmodified $\mathrm{O}$ antigens. For instance, most of the antibody response to $\mathrm{OafA}^{+}$S. typhimurium is directed to O-acetylated (O5) abequose (18). Mice immunized with an $\mathrm{Oaf}^{-}$mutant are more resistant to that mutant than to an isogenic $\mathrm{Oaf}^{+}$strain, and vice versa (18). It is also conceivable that antigenic variations in the LPS may be advantageous to the Salmonella that are actively infecting a host and so are under attack by antibodies to LPS, but there is no evidence that bacteria without modifying enzymes are less virulent in mice. The only known example of lysogeny increasing the virulence of Salmonella involves phage 14 in S. choleraesuis (19). This phage increases polymerization of the repeating LPS units, creating much longer LPS side chains, which makes the bacteria resistant to serum bactericidal activity and hence more virulent in mice. Increasing the chain length of LPS in S. enteritidis also increases its virulence, but the genetic mechanism controlling that process is not known. Because LPS also provides the attachment site for the phage, lysogenized strains that express phage-encoded enzymes are sufficiently altered in their LPS structure that they are not susceptible to superinfection.

Although the lipid A that is attached to the LPS core is conserved in Salmonella, it has recently been shown that the enzymes that make the lipid substitutions on the core galactosamines are regulated in Salmonella by the two component regulator PhoP/Q (20). Mutants that are constitutively on $\left(\mathrm{Pho}^{\mathrm{c}}\right)$ and constitutively off $\left(\mathrm{PhoP}^{-}\right)$are both avirulent in mice. The $\mathrm{Pho}^{\mathrm{c}}$ mutant adds an amino-arabinose to the core disaccharide, hydroxylates a myristic acid, and adds a palmitate to 
one of the amino-galactose core sugars (20). The PhoP strain cannot make these lipid A modifications. Salmonella with the modified LPS are both more resistant to antimicrobial peptides and their lipid A is less stimulatory for macrophages (20).

Flagellin. Another potent antigen on Salmonella is flagellin. Interestingly, most Salmonella-specific CD $4^{+}$ $\mathrm{T}$ lymphocytes that are generated in response to Salmonella infection are directed at flagellin epitopes (21). There is a great deal of variation in the central portion of the flagellin genes, while the $\mathrm{NH}_{2}$ and $\mathrm{COOH}$ termini are highly conserved. This variation is used to define specific flagellar antigens, which are used in the Kauffmann-White scheme to serotype Salmonella. It is not known whether the variations in flagellin structure affect virulence.

Most Salmonella have two sets of flagellin genes that are distinct, and they switch expression between the two at a rate of $10^{-3}$ to $10^{-5}$, so that only one allele is expressed by any given bacterium. The operon that controls the synthesis of phase 1 flagella also encodes a repressor of phase 2 flagellin synthesis (22). The switch to phase 2 flagellin is controlled by a gene that promotes an inversion of a segment of DNA in the phase 1 operon that prevents transcription of the operon, shutting off synthesis of both phase 1 flagellin and the repressor of the phase 2 operon (22). It is possible that this switching mechanism may be a way for the bacteria to temporarily avoid cellular immunity. While antibody to flagellin is not protective, flagellin may be an important T-cell target.

In addition to being antigenic, flagellae from both $S$. typhi and S. typhimurium also stimulate macrophages to make TNF- $\alpha$ (23). Surprisingly, the phase 2 flagellin from $S$. typhimurium is not as potent as the phase $1 \mathrm{fla}$ gellin. This suggests that flagellar phase variation may be a mechanism to downregulate inflammation in the host. The inflammatory potential of individual flagellin proteins made by different Salmonella serotypes has not been extensively compared.

Fimbriae. Salmonella make several classes of fimbriae, including the so-called long polar fimbriae (Lpf), which mediate attachment of Salmonella to Peyer's patches in the mouse. Expression of Lpf in Salmonella undergoes phase variation, such that the bacteria alternate between expressing and not expressing Lpf. Norris and Bäumler recently provided the first demonstration of the biological importance of this phase variation. These authors showed that the lpf genes in S. typhimurium and S. enteritidis are highly conserved and Lpf proteins are antigenically crossreactive (24). Furthermore, while immunization with an Lpf-expressing strain of $S$. typhimurium did not protect against a subsequent challenge with $S$. enteritidis, it did select for phase variants of S. enteritidis not expressing Lpf. This demonstrates the advantage to the pathogen of being able to switch off synthesis of immunogenic surface proteins, and it also suggests why Salmonella have so many different and apparently redundant fimbriae.

\section{Genetic organization of virulence genes}

Pathogenicity islands. A number of crucial virulence phenotypes in Salmonella have been mapped to pathogenicity islands, extended regions of DNA that appear to have inserted in the chromosome although they lack sequence features of known insertional genetic elements such as transposons, lysogenic phage, or integrated plasmids. Salmonella pathogenicity islands (SPIs) are defined as DNA segments encoding virulence genes that are absent from the corresponding region of the $E$. coli $\mathrm{K} 12$ genome sequence, which is approximately colinear with the S. typhimurium chromosome. E. coli and Salmonella appear to have diverged on the order of 100 million years ago. The acquisition of key SPI regions is postulated to have played a major role in this divergence and the adaptation of Salmonella lineages to new niches in their vertebrate hosts.

SPI- 1 was identified as a $40 \mathrm{~kb}$ DNA region inserted in the corresponding E. coli sequence at centisome 63 of the circular genomic map (25). Extensive studies have shown that this region encodes a type III secretion system that transports bacterial proteins into the cytosol of host cells, resulting in cytoskeletal rearrangements that mediate uptake of the Salmonella into a membranebound vesicle (26). While Salmonella can enter many types of cells in tissue culture, the major role of SPI-1 during Salmonella infection is postulated to be in mediating invasion of intestinal epithelial cells. Mutations in the SPI-1 secretion apparatus reduce virulence in oral models of infection but retain virulence when administered systemically. SPI- 1 is present in all known lineages of Salmonella but has not been found in E. coli isolates. This distribution suggests that the acquisition of SPI-1 was a fundamental step in the divergence of these two genera. SPI-1-mediated invasion allowed Salmonella access to a new niche in the intestine: the intracellular environment of intestinal epithelial cells. Deletions in the SPI-1 region have been found in some environmental isolates of certain Salmonella serovars, but all diseaseassociated isolates of the same serovar contain an intact SPI-1 (27). These results suggest that genetic variation in the SPI-1 region occurs, but that disease-producing strains have a functional SPI-1 locus.

The SPI-2 locus was discovered independently by a genome comparison approach (28) and by using signature-tagged mutagenesis (27). This island has subsequently been characterized as a $40 \mathrm{~kb}$ region encoding a second type III secretion system. In contrast to SPI-1, the phenotype of SPI-2 mutants is profound attenuation of virulence in systemic models of infection. On a cellular level, SPI-2 is required for bacterial growth in epithelial cells and for survival in macrophages. SPI-2 
components affect phagosome sorting and the recruitment of the NADPH oxidase to the phagosome membrane $(30,31)$. Thus, SPI- 2 is involved in modifying the intracellular environment encountered by Salmonella.

The SPI-2 locus appears to be a mosaic of regions acquired at different times. Significantly, the type III secretion and effector genes are absent in the S. bongori lineage, but are present in all other Salmonella (32). As noted above, $S$. bongori had been classified separately from the rest of the $S$. enterica lineages as a distinct species and is found principally in cold-blooded vertebrates and the environment. The functions of SPI-2 suggest strongly that it was acquired after SPI-1, since genes related to modifying the intracellular environment would have selective value for an invasive pathogen, but not an organism confined to the intestinal lumen. SPI-2 genes enabled S. enterica lineages to establish a new niche as an intracellular pathogen in the intestinal mucosa and systemic tissues.

The SPI-3 island consists of $17 \mathrm{~kb}$ inserted at the selC tRNA locus (33). The only gene in SPI-3 so far identified with a virulence phenotype is $m g t C$, a gene required for growth in macrophages and systemic virulence in mice. $m g t C$ appears to be required for adaptation to the low- $\mathrm{Mg}^{2+}$ and low $\mathrm{pH}$ conditions found in the intracellular vacuolar environment. While the SPI-3 region displays a mosaic pattern of phylogenetic distribution in the Salmonella lineages, the $m g t C$ locus is present in all lines including $S$. bongori, suggesting that $m g t C$ is essential for organisms that invade host cells. Significantly, an $m g t C$ homologue is present in M. tuberculosis and is also essential for virulence in this unrelated intracellular pathogen (34).

The phylogenetic distributions of two other putative pathogenicity islands, designated SPI-4 and SPI-5, have not been reported, nor have the roles of these regions in Salmonella pathogenesis been completely established. The designation of SPI- 4 as a pathogenicity island rests on the phenotype of a single transposon insertion previously reported to decrease macrophage survival (35). SPI- 5 was identified as a region surrounding the $\operatorname{sop} B$ gene in S. dublin (36). The DNA sequence of SPI-5 contains six putative genes, and mutations in four of these $(\operatorname{sop} B$, $p i p A, p i p B$, and $p i p D)$ have a similar phenotype: decreased fluid accumulation and inflammation in ligated bovine ileal loops and normal virulence in systemic infections of mice. The $\operatorname{sop} B$ gene encodes an inositol phosphate phosphatase that is transported into epithelial cells by the SPI- 1 secretion system and indirectly affects chloride secretion by inositol phosphate signaling pathways $(37,38)$. However, the role of $S o p B$ in enteric disease is unclear, since a $\operatorname{sop} B$ mutation of $S$. typhimurium produced enteritis and mortality in calves equivalent to the wild-type bacteria (39).

The spv genes. Another set of genes that profoundly influence Salmonella virulence are found in an operon designated $s p v$ (originally an abbreviation for Salmonella plasmid virulence). These genes were first discovered on large $(50-100 \mathrm{~kb})$ plasmids in certain serovars of the subspecies I lineage (40). All mouse-virulent strains of Salmonella contain virulence plasmids, and the highly conserved spv locus on these plasmids is both necessary and sufficient for the plasmid-mediated murine virulence phenotype. Virulence plasmids are found in serovars that are host-adapted to domestic animals: dublin (cattle), choleraesuis (pigs), gallinarum/pullorum (fowl), abortusovis (sheep), as well as the two common, broad-host-range serovars, typhimurium and enteritidis. Recently, the $s p v$ locus has also been found in most of the other Salmonella subspecies (41). However, in these lineages, the spv genes appear to be located in the chromosome, and the roles of the chromosomal spv loci in the virulence of these subspecies have not been determined.

The sp $v$ locus consists of five genes: the positive regulatory gene $s p v R$ and four structural genes, $s p v A, s p v B$, $s p v C$, and $s p v D(40)$. Mutations in $s p v R$ abolish expression of all the spv genes and have been used to define the Spv phenotype. In mice, the spv genes do not affect initial intestinal colonization or invasion, but $s p v$ mutants are profoundly attenuated for systemic infection. The effect of the spv genes on Salmonella infection of tissue culture cells has been difficult to define, but recent evidence indicates that the spv locus is required for bacterial growth in monocyte-derived macrophages (MDM) of both bovine and human origin $(42,43)$. In human MDM, the major $s p v$ phenotype appears to be induction of cytotoxicity manifested by detachment and eventual apoptosis of infected cells accompanied by intracellular bacterial proliferation (43).

Conflicting results have been reported on the role of the spv genes in enteric disease. In mice - a poor model for intestinal disease, since they do not develop enteritis in response to Salmonella infection - the presence of the $s p v$ genes does not affect the numbers of bacteria recovered from the bowel wall or Peyer's patch tissue. In contrast, calves develop severe enteritis after oral inoculation of S. typhimurium or S. dublin. An spvR mutant of $S$. typhimurium causes lethal enteric infection in calves similar to the wild-type strain (39). One study (42) reported attenuation of both enteric and systemic disease with an $s p v R$ mutant of S. dublin, a serovar host-adapted to cattle, but another group (44) found that the spv genes only affect systemic virulence in $S$. dublin. Taken together, the evidence indicates that the $s p v$ genes facilitate systemic virulence in $S$. enterica subspecies I serovars that naturally contain virulence plasmids, and that the spv effect on enteric disease may be variable depending on the specific strains and hosts involved. For human infection, epidemiologic evidence suggests that the spv genes promote dissemination of S. typhimurium from the intestine (45).

Islets and individual genes. A number of studies have identified small genetic loci (termed islets) or indi- 
vidual genes that encode virulence traits and that are variably present in different Salmonella lineages or individual strains. The sopE gene, which encodes an accessory epithelial cell invasion factor, is present on a temperate bacteriophage which is carried by some isolates of S. typhimurium and certain other serovars in subspecies I (46). Likewise, Salmonella strains differ in the presence of the sodCI gene, encoding one of the two periplasmic superoxide dismutase enzymes (47). sodCI is found in the nontyphoid serovars that cause the majority of human bacteremias. The other gene, sodCII, is present in all Salmonella tested and is highly homologous to the E. coli sodC. A gene designated shdA is associated with fecal shedding and is present in subspecies I strains but absent from the other subspecies, suggesting that $s b d A$ influences adaptation to the intestine of warm-blooded animals (48).

\section{Summary}

Salmonella strains have evolved to infect a wide variety of reptiles, birds, and mammals resulting in many different syndromes ranging from colonization and chronic carriage to acute fatal disease. Adaptation to a large number of different evolutionary niches has undoubtedly driven the high degree of phenotypic and genotypic diversity in Salmonella strains. Differences in LPS and flagellar structure generate the antigenic variation that is reflected in the more than 2,000 known serotypes. Moreover, variations of LPS structure affect the virulence of the strain. The differential expression of various fimbriae by Salmonella is likely to be due to the wide variety of mucosal surfaces that are encountered by various strains, and the host immune response may select for a different expression pattern. As with these surface structures, a variety of other important virulence determinants show a variable distribution in Salmonella strains and also serve to delineate the divergence of the Salmonella lineage from E. coli. The acquisition of the SPI-1 region may have represented the defining genetic event in the separation of the Salmonella and E. coli lineages. The SPI- 1 cell invasion function allowed Salmonella to establish a separate niche in epithelial cells. The $m g t C$ locus on SPI- 3 is also present in all lineages and facilitates the adaptation of the bacteria to the low $\mathrm{Mg}^{2+}$, low $\mathrm{pH}$ environment of the endosome that results from SPI-1-mediated invasion. Subsequent acquisition of SPI-2 allowed Salmonella to manipulate the sorting of the endosome or phagosome, altering the intracellular environment and facilitating bacterial growth within infected cells. The ability to disseminate from the bowel and establish extraintestinal niches is promoted by the spv locus. Since Salmonella proliferates within macrophages and must avoid phagocytosis by neutrophils to establish a systemic infection (49), the spv genes appear to promote the macrophage phase of the disease process.
Here the polymorphism of the spv locus is clearly demonstrated, since the serovars that cause most cases of nontyphoid bacteremia contain the spv genes. The absence of the spv genes from $S$. typhi is particularly puzzling (40) and is a strong indication that the pathogenesis of typhoid fever is fundamentally different from that of bacteremia due to nontyphoid Salmonella. There is currently no genetic explanation for the phenotype of host adaptation or for the finding that only a few serovars cause the majority of human infections. Based on recent findings that multiple individual virulence genes have a variable distribution in Salmonella, it is unlikely that a single locus will be found to be responsible for these complex biological traits (50). Instead, a complicated combination of genes are likely to contribute to the overall virulence phenotype.

1. Fierer,J., and Swancutt, M. 2000. Non-typhoid Salmonella: a review. Curr. Clin. Top. Infect. Dis. 20:134-157.

2. Le Minor, L. 1988. Typing of Salmonella species. Eur. J. Clin. Microbiol. Infect. Dis. 7:214-218.

3. Blaser, M.J., and Feldman, R.A. 1981. Salmonella bacteremia: reports to the Centers for Disease Control, 1968-1979. J. Infect. Dis. 143:743-746.

4. Donnenberg, M.S., and Whittam, T.S. 2001. Pathogenesis and evolution of virulence in enteropathogenic and enterohemorrhagic Escherichia coli.J. Clin. Invest. 107:539-548.

5. Liu, D., Verma, N.K., Romana, L.K., and Reeves, P.R. 1991. Relationships among the $r f b$ regions of Salmonella serovars A, B, and D. J. Bacteriol. 173:4814-4819.

6. Bock, K., et al. 1984. The conformation of Salmonella O-antigenic polysaccharide chains of serogroups $\mathrm{A}, \mathrm{B}$, and $\mathrm{D}_{1}$ predicted by semi-empirical, hardsphere (HSEA) calculations. Carbohydr. Res. 130:23-34.

7. Joiner, K.A., Hammer, C.H., Brown, E.J., and Frank, M.M. 1982. Studies on the mechanism of bacterial resistance to complement-mediated killing. II. C8 and C9 release C5b67 from the surface of Salmonella minnesota S218 because the terminal complex does not insert into the bacterial outer membrane. J. Exp. Med. 155:809-819.

8. Jimenez-Lucho, V.E., Joiner, K.A., Foulds, J., Frank, M.M., and Leive, L. 1987. $\mathrm{C} 3 \mathrm{~b}$ generation is affected by the structure of the $\mathrm{O}$-antigen polysaccharide in lipopolysaccharide from salmonellae. J. Immunol. 139:1253-1259.

9. Liang-Takasaki, C.-J., Makela, P.H., and Leive, L. 1982. Phagocytosis of bacteria by macrophages: changing the carbohydrate of lipopolysaccharide alters interaction with complement and macrophages. J. Immunol. 128:1229-1235.

10. Johnson, B.N., Weintraub, A., Lindberg, A.A., and Stocker, B.A.D. 1992. Construction of Salmonella strains with both antigen O4 (of group B) and antigen O9 (of group D). J. Bacteriol. 174:1911-1915.

11. Cygler, M., Rose, D.R., and Bundle, D.R. 1991. Recognition of a cell-surface oligosaccharide of pathogenic Salmonella by an antibody fab fragment. Science. 253:442-445.

12. Mittrücker, H.-W., and Kaufmann, S.H.E. 2000. Immune response to infection with Salmonella typhimurium in mice. J. Leukoc. Biol. 67:457-463.

13. Plotkin, S.A., and Bouveret-Le Cam, N. 1995. A new typhoid vaccine composed of the Vi capsular polysaccharide. Arch. Intern. Med. 155:2293-2299.

14. Saxen, H. 1984. Mechanism of the protective action of anti-Salmonella IgM in experimental mouse salmonellosis. J. Gen. Microbiol. 130:2277-2283.

15. Carlin, N.I.A., Svenson, S.B., and Lindberg, A.A. 1987. Role of monoclonal $\mathrm{O}$-antigen antibody epitope specificity and isotype in protection against experimental mouse typhoid. Microb. Pathog. 2:171-183.

16. Fierer,J., Chikami, G., Hatlen, L., Heffernan, E.J., and Guiney, D. 1988. Active immunization with LD842, a plasmid-cured strain of Salmonella dublin, protects mice against group D and group B Salmonella infection. J. Infect. Dis. 158:460-463.

17. Slauch, J.M., Lee, A.A., Mahan, M.J., and Mekalanos, J.J. 1996. Molecular characterization of the oafA locus responsible for acetylation of Salmonella typhimurium $\mathrm{O}$-antigen: OafA is a member of a family of integral membrane trans-acylases. J. Bacteriol. 178:5904-5909.

18. Kim, M.L., and Slauch, J.M. 1999. Effect of acetylation (O-factor 5) on the 


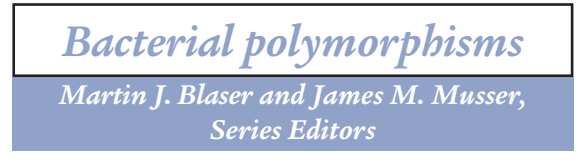

polyclonal antibody response to Salmonella typhimurium O-antigen. FEMS Immunol. Med. Microbiol. 26:83-92.

19. Nnalue, N.A., Newton, S., and Stocker, B.A.D. 1990. Lysogenization of Sal monella choleraesuis by phage 14 increases average length of O-antigen chains, serum resistance and intraperitoneal mouse virulence. Microb. Pathog. 8:393-402.

20. Guo, L., et al. 1997. Regulation of lipid a modifications by Salmonella typhimurium virulence genes phoP-phoQ. Science. 276:250-253.

21. Cookson, B.T., and Bevan, M.J. 1997. Identification of a natural T cell epitope presented by Salmonella-infected macrophages and recognized by $\mathrm{T}$ cells from orally immunized mice. J. Immunol. 158:4310-4319.

22. Kutsukake, K., and Iino, T. 1980. A trans-acting factor mediates inversion of a specific DNA segment in flagellar phase variation of Salmonella. Nature. 284:479-481.

23. Ciacci-Woolwine, F., Blomfield, I.C., Richardson, S.H., and Mizel, S.B. 1998. Salmonella flagellin induces tumor necrosis factor alpha in a human promonocytic cell line. Infect. Immun. 66:1127-1134.

24. Norris, T.L., and Bäumler, A.J. 1999. Phase variation of the Ipfoperon is a mechanism to evade cross-immunity between Salmonella serotypes. Proc. Natl. Acad. Sci. USA. 96:13393-13398.

25. Mills, D.M., Bajaj, V., and Lee, C.A. 1995. A 40 kilobase chromosomal fragment encoding Salmonella typhimurium invasion genes is absent from the corresponding region of the Escherichia coli K-12 chromosome. Mol. Microbiol. 15:749-759.

26. Galan, J.E. 1999. Interaction of Salmonella with host cells through the centosome 63 type III secretion system. Curr. Opin. Microbiol. 2:46-50.

27. Ginocchio, C.C., Rahn, K., Clarke, R.C., and Galan, J.E. 1997. Naturally occurring deletions in the centisome 63 pathogenicity island of environmental isolates of Salmonella spp. Infect. Immun. 65:1267-1272.

28. Ochman, H., Soncini, F.C., Solomon, F., and Groisman, E.A. 1996. Identification of a pathogenicity island required for Salmonella survival in host cells. Proc. Natl. Acad. Sci. USA. 93:7800-7804.

29. Shea, J.E., Hensel, M., Gleeson, C., and Holden, D.W. 1996. Identification of a virulence locus encoding a second type III secretion system in Salmonella typhimurium. Proc. Natl. Acad. Sci. USA. 93:2593-2597.

30. Vchiya, K., et al. 1999. A Salmonella virulence protein that inhibits cellular trafficking. EMBO J. 18:3924-3933.

31. Vazquez-Torres, A., et al. 2000. Salmonella pathogenicity island 2-dependent evasion of the phagocyte NADPH-oxidase. Science. 287:1655-1658.

32. Hensel, M., Nikolaus, T., and Egelseer, C. 1999. Molecular and functional analysis indicates a mosaic structure of Salmonella pathogenicity island 2. Mol. Microbiol. 31:489-498.

33. Blanc-Potard, A.-B., and Groisman, E.A. 1997. The Salmonella selC locus contains a pathogenicity island mediating macrophage survival. EMBO J. 16:5376-5385.

34. Buchmeier, N., et al. 2000. A parallel intraphagosomal survival strategy shared by mycobacterium tuberculosis and Salmonella enterica. Mol. Microbiol. 35:1375-1382.

35. Wong, K.-K., et al. 1998. Identification and sequence analysis of a 27-kilo- base chromosomal fragment containing a Salmonella pathogenicity island located at 92 minutes on the chromosome map of Salmonella enterica serovar typhimurium LT2. Infect. Immun. 66:3365-3371.

36. Wood, M.W., et al. 1998. Identification of a pathogenicity island required for Salmonella enteropathogenicity. Mol. Microbiol. 29:883-891.

37. Eckmann, L., et al. 1997. D-myo-Inositol 1,4,5,6-tetrakisphosphate produced in human intestinal epithelial cells in response to Salmonella invasion inhibits phosphoinositide 3-kinase signaling pathways. Proc. Natl. Acad. Sci. USA. 94:14456-14460.

38. Norris, F.A., Wilson, M.P., Wallis, T.S., Galyov, E.E., and Majerus, P.W. 1998. SopB, a protein required for virulence of Salmonella dublin, is an inositol phosphate phosphatase. Proc. Natl. Acad. Sci. USA. 95:14057-14059.

39. Tsolis, R.M., Adam, G., Ficht, T.A., and Baumler, A.J. 1999. Contribution of Salmonella typhimurium virulence factors to diarrheal disease in calves. Infect. Immun. 67:4879-4885.

40. Guiney, D.G., et al. 1995. Biology and clinical significance of virulence plasmids in Salmonella serovars. Clin. Infect. Dis. 21(Suppl.):S146-S151.

41. Boyd, E.F., and Hartl, D.L. 1998. Salmonella virulence plasmid: modular acquisition of the $s p v$ virulence region by an F-plasmid in Salmonella enterica subspecies I and insertion into the chromosome of subspecies II, IIIa, IV and VII isolates. Genetics. 149:1183-1190.

42. Libby, S.J., et al. 1997. The spv genes on the Salmonella dublin virulence plasmid are required for severe enteritis and systemic infection in the natural host. Infect. Immun. 65:1786-1792.

43. Libby, S.J., Lesnick, M., Hasegawa, P., Weidenhammer, E., and Guiney, D.G. 2000. The Salmonella virulence plasmid spv genes are required for cytopathology in human monocyte-derived macrophages. Cell. Microbiol. 2:49-58.

44. Wallis, T.S., Paulin, S.M., Plested, J.S., Watson, P.R., and Jones, P.W. 1995. The Salmonella dublin virulence plasmid mediates systemic but not enteric phases of salmonellosis in cattle. Infect. Immun. 63:2755-2761.

45. Fierer, J., Krause, M., Tauxe, R., and Guiney, D. 1992. Salmonella typhimurium bacteremia: association with the virulence plasmid. J. Infect. Dis. 166:639-642.

46. Mirold, S., et al. 1999. Isolation of a temperate bacteriophage encoding the type III effector protein SopE from an epidemic Salmonella typhimurium strain. Proc. Natl. Acad. Sci. USA. 96:9845-9850.

47. Fang, F.C., et al. 1999. Virulent Salmonella typhimurium has two periplasmic Cu, Zn-superoxide dismutases. Proc. Natl. Acad. Sci. USA. 96:7502-7507.

48. Kingsley, R.A., van Amsterdam, K., Kramer, N., and Baumler, A.J. 2000. The $s h d A$ gene is restricted to serotypes of Salmonella enterica subspecies I and contributes to efficient and prolonged fecal shedding. Infect. Immun. 68:2720-2727.

49. Vassiloyanakopoulos, A.P., Okamoto, S., and Fierer, J. 1998. The crucial role of polymorphonuclear leukocytes in resistance to Salmonella dublin infections in genetically susceptible and resistant mice. Proc. Natl. Acad. Sci. USA. 95:7676-7681.

50. Lu, S., Manges, A.R., Xu, Y., Fang, F.C., and Riley, L.W. 1999. Analysis of virulence of clinical isolates of Salmonella enteritidis in vivo and in vitro. Infect. Immun. 67:5651-5657. 